\title{
Close-up on cell biology
}

\section{To define the workings of cellular structures and molecules requires cutting-edge technology not only in biology and biochemistry, but also now in nanotechnology. Hayley M. Birch and Julie Clayton report.}

The dawn of the nano-era has placed a whole new array of tools in the hands of cell biologists who are keen to go deeper into the intricacies of how cells work. Forever pushing the boundaries, cell biologists are shifting focus from the micro- towards the nano- and even sub-nano level. To do so means having to find ever more creative ways of using not just biology, but also elements of physics, materials science and engineering. Biophysicists, nanotechnologists, nanofabricators and electrical engineers are working side by side to probe further into the cell.

\section{Nanotopography}

Knowing what makes a cell tick is as much about understanding its outside environment as its internal workings. The availability of sophisticated nanopatterning and nanotopography techniques - in which surfaces can be etched and coated with a variety of substances at the nanoscale - is enabling cell biologists to gain a more precise understanding of, and control over, cellular responses compared with cell culture in conventional glass or polystyrene culture plates A range of substrate structures designed

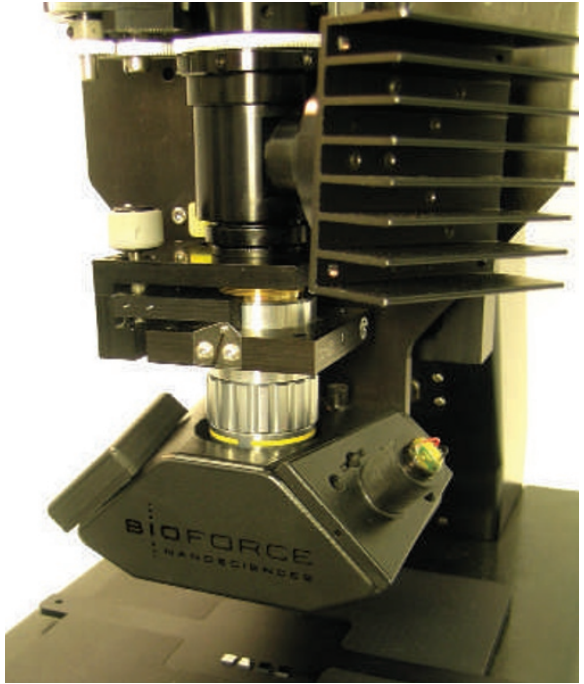

The Nano eNabler deposits molecules at nanometre resolution.

specifically for exploring cell behaviours such as spreading, migration and adhesion are under development (see "Cell culture in three dimensions" and "Down to the letter", page 940).
The field is so new that most research teams are creating their own nanopatterned surfaces on which the cells are cultured. At Columbia University, New York, Michael Sheetz is developing nanopatterning methods with a team of cell biologists, systems biologists and nanofabricators. "We put down arrays of different spacings of molecules and use those to measure cellular responses," he explains. "We have found that the spacing really matters." Sheetz and colleagues are currently using patterning to examine how the spacing of protein ligands on a substrate surface is important in the binding of dimeric proteins such as talin.

While Sheetz and many others are currently using home-made devices, commercial developers are beginning to sense an opportunity. BioForce Nanoscience in Ames, Iowa, has developed the Nano eNabler, a device that can deposit molecules on surfaces at defined locations at nanometre resolution, and which, among its various options, can be used to apply extracellular matrix proteins onto cell-culture surfaces in predefined patterns. A key feature of the machine is that the drops deposited can be as large as $100 \mu \mathrm{m}$ or as small as $2 \mu \mathrm{m}$

\section{CELL CULTURE IN THREE DIMENSIONS}

Taking the idea of nanoscale design for cell culture further, some cell biologists are investigating cellular responses to more complex threedimensional structures.

A collaboration in this field between cell biologists and electronic engineers at the University of Glasgow's Centre for Cell Engineering is proving fruitful.

The group has the advantage of a stateof-the-art electronbeam nanolithography system (E Beam)

deliberately," says Mathis Riehle, who directs research at the centre. "With the E Beam you can be very specific."

The $E$ Beam is used to define or

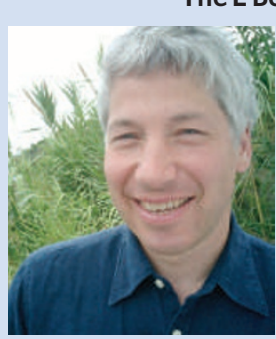

Mathis Riehle: "With the $\mathrm{E}$ Beam you can be very specific."

manufactured by Leica, which creates nanoscale features on a surface under the control of customized software (see also 'Down to the letter'). "We're quite unique in the United Kingdom in that we have access to such an incredible machine with which you can define structures so "write" a pattern at nanometric resolution into an electronsensing polymer. The pattern is developed and used as a template for etching or depositing material to form the desired nanotopographic features. This technique gives a greater degree of control than 'natural' lithography, which relies on self-assembly of colloidal particles in regular arrays to produce the pattern.

Riehle wants to explore the limits of the machine. "We've made tubes and we want to make more complicated three-dimensional structures - maybe structures that will look like cell 'car parks'. But to do that we would need something like origami, because at the moment the E Beam can only really write on a flat plane. We cannot write on a shaped, undulating surface." The team will have to write its own software to create these complex structures.

As one of a consortium of institutions working on emerging nanopatterning methods, the Glasgow centre is also developing alternative methods of engineering 3D structures with nanoscale features for use as cell-culture substrates. Kris Seunarine and Osian Meredith have fabricated a 'swiss roll' from e-polycaprolactone, a biodegradable thermoplastic shaped by hot nano- and micro- embossing. This is the 'sponge' of the swiss roll, with the cells as the 'jam'.

Two levels of microfeatures are sculpted into the polymer (see photos). One aligns fibroblasts and smooth-muscle cells, keeping them from being squashed. The inwardfacing surface is patterned at the nanoscale with a regular array of pits $(100 \mathrm{~nm}$ diameter and $80 \mathrm{~nm}$ deep) spaced $300 \mathrm{~nm}$ apart, which define locations for endothelial cell adhesion. These cell scaffolds could be useful in developing methods for vascular and urogenital reconstruction.

H.M.B.

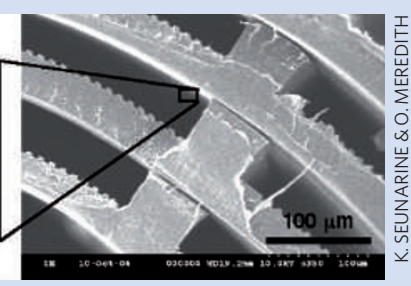

Cell support: ridges on the surface of the 'swiss roll' will keep cells aligned, and pits provide anchorage. 